\title{
AN AGILE TIP-TILT-PISTON MICROMIRROR WITH LARGE APERTURE, LARGE SCANNING RANGE AND LOW DRIVING VOLTAGE
}

\author{
Kemiao Jia, Sagnik Pal, and Huikai Xie \\ Department of Electrical and Computer Engineering, University of Florida, Gainesville, FL 32611, USA
}

\begin{abstract}
This paper presents the design, fabrication and test results of an electrothermally-actuated tip-tilt-piston micromirror with a large optical aperture size of $1 \mathrm{~mm}$. The mirror has a mechanical rotation angle of $\pm 6^{\circ}$ about both $\mathrm{x}$ - and $\mathrm{y}$ - axis and displaces $141 \mu \mathrm{m}$ in z-axis all at dc voltages of less than $6 \mathrm{~V}$. Dynamic test of the micromirror shows the electrothermal response time of each actuator is about $20 \mathrm{~ms}$. The resonant frequencies of the piston and rotation motion are $664 \mathrm{~Hz}$ and $714 \mathrm{~Hz}$, respectively. The large tip-tilt-piston scanning capability at low driving voltage makes this type of devices suitable for biomedical imaging and laser beam steering applications.
\end{abstract}

\section{INTRODUCTION}

MEMS micromirrors have been widely used in various applications including optical communications, displays, medical imaging, and motion tracking [1]-[9]. For adaptive optics, optical phase arrays, and optical phase modulators, micromirrors with tip-tilt-piston (TTP) capability are required, which have been demonstrated using electrostatic, electrothermal and piezoelectric actuation [10]-[14][16][18]. Among various actuation mechanisms, electrothermal actuators usually are not very fast and consume relatively high power. On the other hand, electrothermal micromirrors produce large scan ranges at low driving voltages, making them very suitable for medical imaging applications, especially endoscopic imaging applications in which operating frequency is not high and power consumption is not critical.

Various bimorph-based electrothermal TTP micromirrors have been demonstrated [14]-[18]. For instance, Jain et al. demonstrated an electrothermal micromirror using a DRIE CMOS-MEMS process [15]. The device generated a maximum piston motion of $0.5 \mathrm{~mm}$ and tip/tilt optical scan angle of $\pm 30^{\circ}$ at less than 15V DC. However, its mirror plate had a large lateral shift in the piston mode and its rotation axes of the tip/tilt modes shifted vertically during scanning. The large lateral shift of the mirror plate significantly reduces the effective optical aperture size, and the rotation-axis shift causes difficulty in optical alignment and image processing. In order to solve the lateral shift problem, Wu et al. developed a three-segment bimorph actuator design that achieved near lateral-shift-free large vertical actuation [16]. But its tip/tilt scan patterns are not horizontal, and the rotation-axis shift, though smaller, still exists. Todd et al. proposed an S-shaped, invertedseries-connected (ISC) bimorph actuator to overcome both mirror plate shift and rotation axis shift problems [17]. The ISC concept is illustrated in Fig. 1. With a single bimorph, as shown in Fig. 1(a), there exists a lateral shift and a tangential tilt angle at the tip of the bimorph. If two bimorphs with opposite layer compositions are connected in series to form an S-shaped beam, as shown in Fig. 1(b), the tilting angle at the tip of the second bimorph is compensated. However, the lateral shift still remains. If two S-shaped beams are connected with the second one inverted, as shown in Fig. 1(c), both the lateral shift and the tilt angle will be compensated. Jia et al. demonstrated the first working TTP micromirror based on this ISC bimorph actuation concept, but the scanning ranges were small and there were problems due to aluminum sidewall pileup and structural asymmetry [18]. Furthermore, the joints of the S-shaped beams in Fig. 1(b) and Fig. 1(c) are fragile.

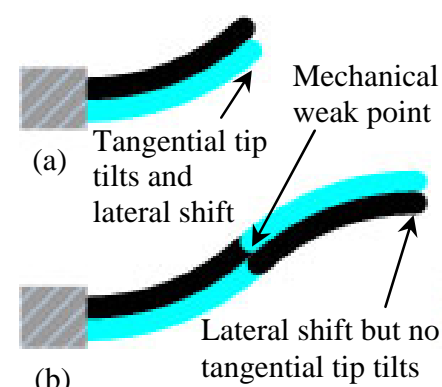

(b) tangential tip tilts

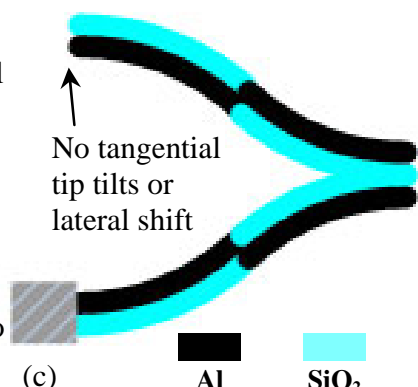

Fig. 1 ISC concept evolution. (a) Single bimorph with lateral shift and tangential tip tilt. (b) Series-connected bimorphs with zero tip tilt but nonzero lateral shift. (c) Inverted-series-connected bimorphs with zero tip tilt and zero lateral shift.

In this paper, we present a single-crystal-silicon based TTP micromirror employing a new ISC bimorph design that solves the above mentioned problems. The design, fabrication and test of the device will be presented and discussed in the following sections.

\section{DEVICE DESIGN AND FABRICATION}

The new ISC bimorph actuator design is illustrated in Fig. 2(a), where the main improvement is adding an overlap at the middle of each S-shaped bimorph beam. The length of the overlap is optimized to approximately $1 / 3$ of the total beam length to achieve maximum initial elevation of the mirror plate. The width of the overlap is widened to strengthen the connection as well as increase the stiffness of the actuator. Also, platinum instead of poly-silicon is used as the heater material. The temperature of the beams can be changed by applying a voltage to the Pt resistors embedded along the bimorph beams. By connecting four pairs of ISC actuators to the four sides of a square or circular mirror plate, tip-tilt-piston operation can be realized by controlling the voltages applied to the resistors. The 2D schematic of the device is shown in Fig. 2(b).

When a same voltage is applied to the four actuators simultaneously, the mirror plate moves vertically without any lateral shift. When different voltages are applied to the actuators, the mirror plate performs tip-tilt scanning. As shown in Fig. 2, point B, which connects a bimorph actuator to the mirror plate, moves pure vertically if the bimorph beam is heated evenly. This condition is achieved by embedding a heater along the entire bimorph path. When a same dc voltage is applied to all the four actuators and a pair of differential ac voltage signals is applied to one pair of opposing actuators, i.e., Act 1 and Act 3 , the mirror plate will rotate about the $y$-axis and the rotation axis is structurally fixed by the other two opposing actuators, i.e., Act2 and Act4. Thereby, both piston motion decoupled from rotation and tip-tilt scanning with a fixed rotation axis can be obtained.

The fabrication process of the device is shown in Fig. 3. It starts from a bare silicon wafer. First, a $1 \mu \mathrm{m}$ thick PECVD $\mathrm{SiO}_{2}$ is deposited and patterned to form the bimorphs that require $\mathrm{SiO}_{2}$ to be the bottom layer (Fig. 3a). Then, the wafer is patterned, followed by a $0.25 \mu \mathrm{m}$ thick Cr-Pt-Cr sputtering and lift-off (Fig. 3b). After a thin $\mathrm{SiO}_{2}$ layer is deposited, aluminum lift-off is performed to define the bimorphs and the mirror plate (Fig. 3c). This step has been modified from the previously reported process to solve the aluminum 
Room temperature

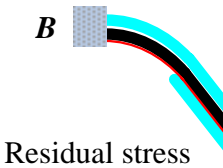

induced initial

elevation

(a)

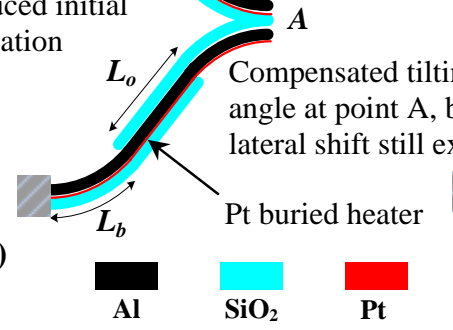

Elevated Temperature

Pure vertical displacement at point $\mathrm{B}$ with no tilting

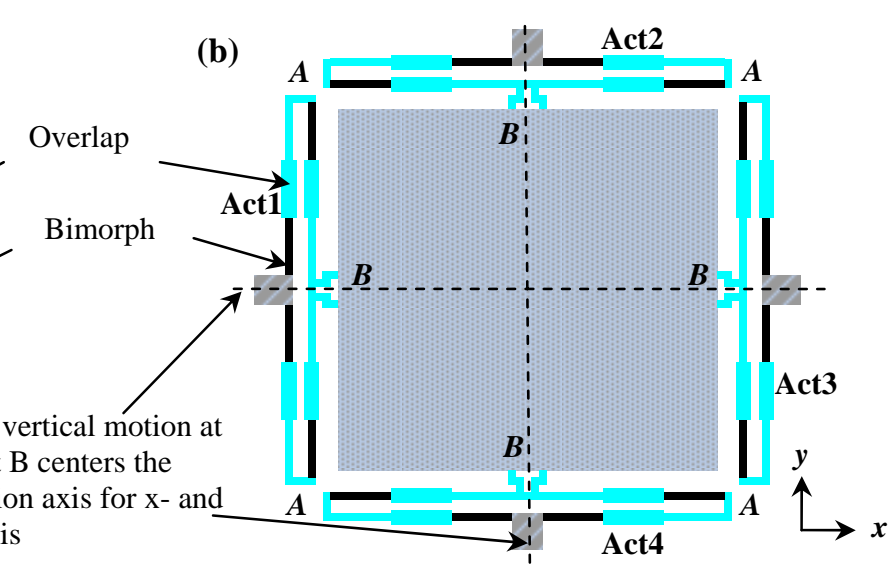

Fig.2 (a) New ISC bimorph actuation concept. (b) Actuator configuration for the tip-tilt-piston mirror with centered rotation axes.

sidewall pileup problem [18]. Next, a second $\mathrm{SiO}_{2}$ layer is deposited and patterned to form the bimorphs that require $\mathrm{SiO}_{2}$ to be the top layer (Fig. 3d). A backside silicon etch is then proceeded to form the cavity under the mirror plate and the actuators, leaving a controllable thickness $(\sim 50 \mu \mathrm{m})$ of silicon to support the mirror plate (Fig. 3e). Finally, a front side silicon etch-through followed by an isotropic undercut is done to release the device (Fig. 3f).

An SEM of a fabricated device is shown in Fig. 4(a), where the mirror plate is $1 \mathrm{~mm}$ by $1 \mathrm{~mm}$ with an initial elevation of $141 \mu \mathrm{m}$. Fig. 4(b) shows some details of a bimorph actuator and mechanical connections. Notice that in Fig. 4(c) a device with the mirror plate below the substrate surface is also obtained by simply swapping $\mathrm{SiO}_{2}$ and $\mathrm{Al}$ in all bimorph segments. This design helps protect the fragile mirror surface for easy handling and packaging and has a similar piston stroke and optical scan range compared with the one in Fig. 4(a). The devices shown here have a fill factor of about $60 \%$.

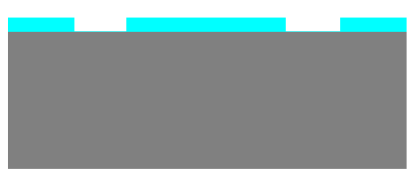

(a)

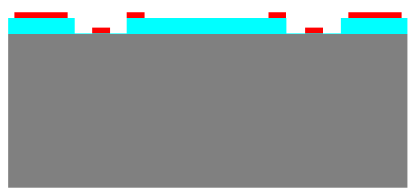

(b)

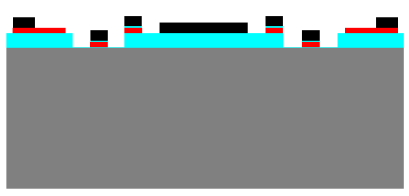

(c)

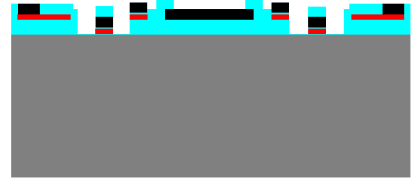

(d)

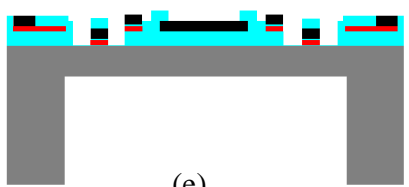

(e)

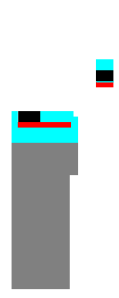

(f)

\section{Al}

Fig. 3 Process flow: (a) Bottom oxide pattern. (b) Cr-Pt-Cr heater lift-off. (c) Insulation oxide deposition and Al lift-off. (d) Top oxide pattern. (e) Backside silicon etch. (f) Front side silicon etch-through and release.

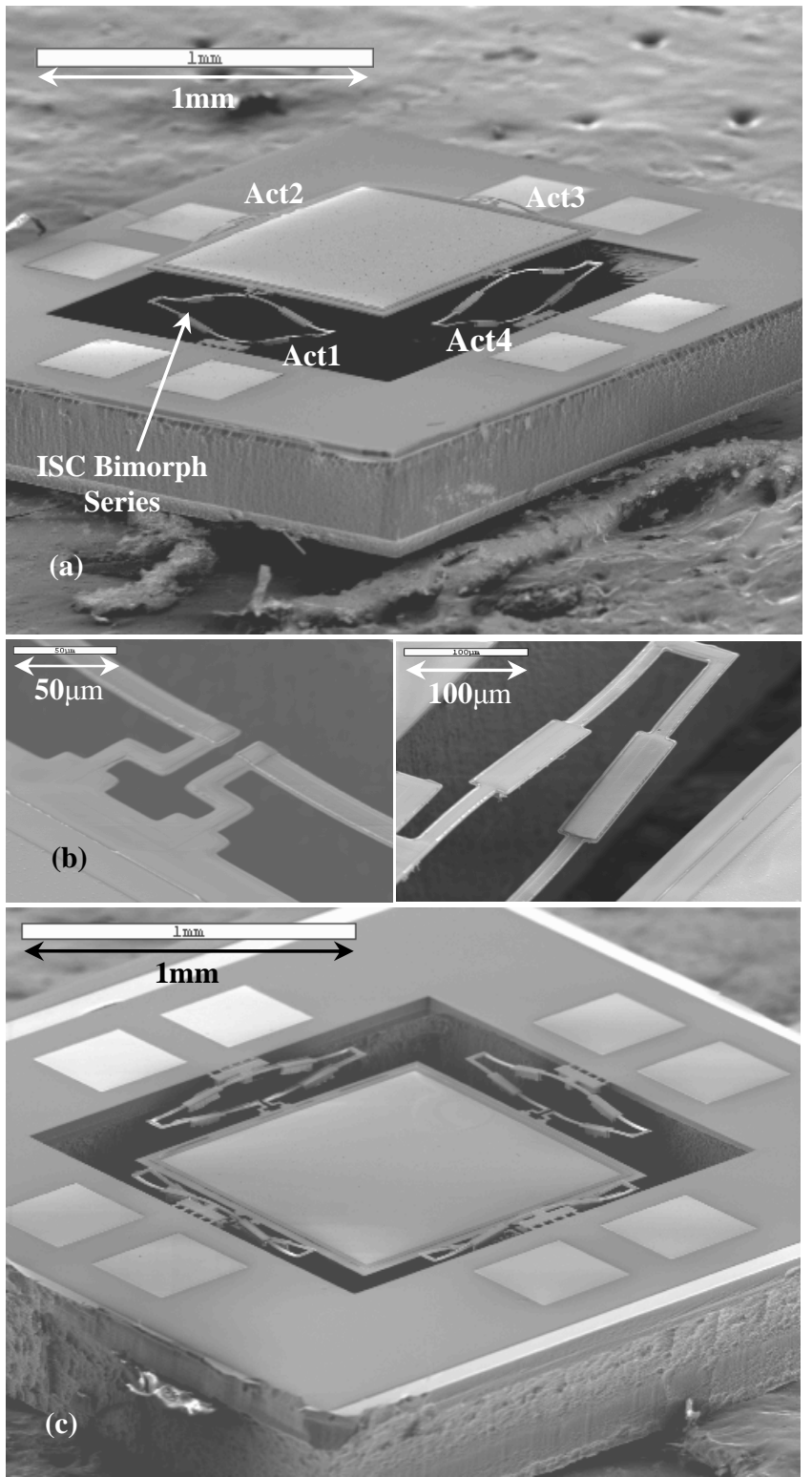

Fig.4 (a) SEM image of a fabricated device. (b) Bimorph series and connections. (c) A device with mirror plate below substrate. 


\section{EXPERIMENTAL RESULTS}

\section{Static Measurement Results}

Piston displacement of the mirror plate is achieved by driving the four actuators simultaneously with a same dc voltage. In this experiment, a wire-bonded device was placed under an Olympus BX51 optical microscope equipped with a Quadra-Chek 200 micro-position recorder to track the vertical displacement. After each increase of the supply voltage, the image was refocused and a reading of $\mathrm{z}$ position of the mirror plate was recorded. Data points for multiple locations on the mirror plate were taken to verify its piston motion. It was found that the tilting of the mirror plate was initially $0.2^{\circ}$ and this tilting did not become larger during the actuation. It was also recorded from this test that the lateral shift of the mirror plate was only $2 \mu \mathrm{m}$ over the entire actuation range of $\sim 140 \mu \mathrm{m}$ at $5 \mathrm{~V}$ dc. The error in this measurement is $\pm 1 \mu \mathrm{m}$ resulting from the refocusing step. Fig. 5 shows the static measurement result for the piston actuation.

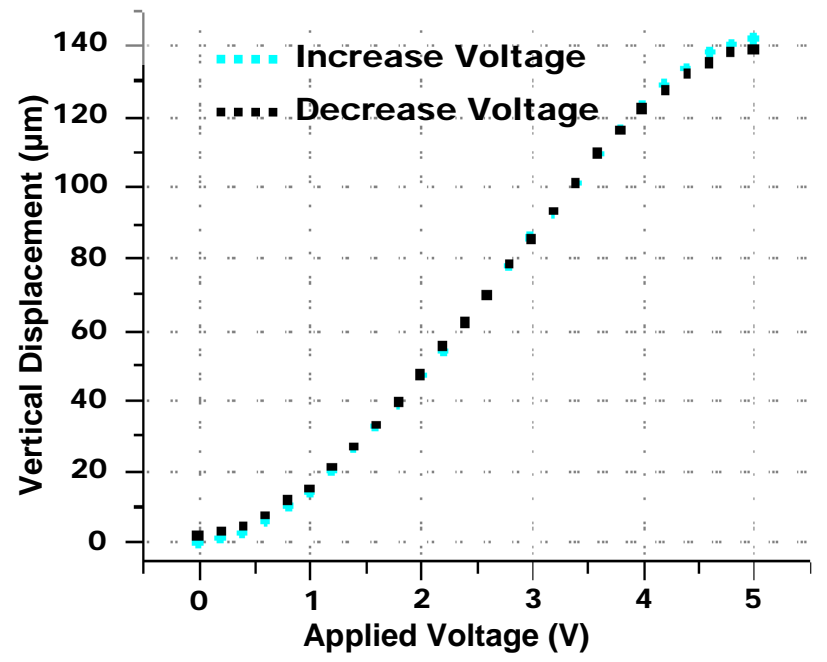

Fig. 5 Static measurement result for piston mode actuation. Same dc voltage was applied to all four actuators.

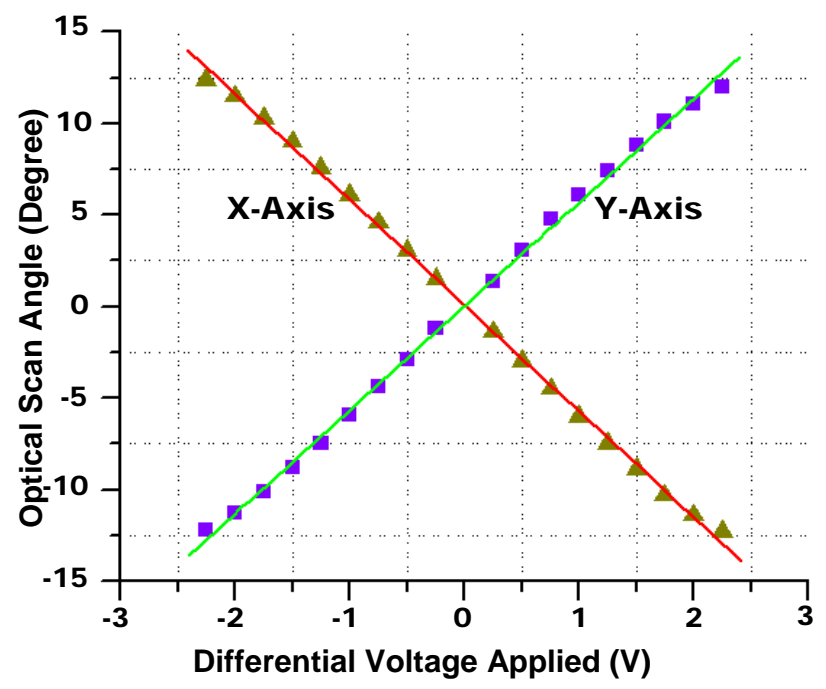

Fig. 6 Static measurement result for rotation mode actuation. All actuators had a dc voltage of 2.25V. Differential voltages were applied to opposing actuator pairs.
The static rotation measurement was taken by applying a same dc voltage of $2.25 \mathrm{~V}$ to all four actuators and at the same time superimposing two differentially varying voltages to one opposing actuator pair. A laser beam and a screen were used for this experiment. Fig. 6 shows the static measurement result for the rotation actuation. An optical scan angle of $\pm 12^{\circ}$ was achieved for both $\mathrm{x}$ - and $\mathrm{y}$-axis at a pair of $2.25 \mathrm{~V}$ differential voltages.

The measured resistances of the heaters are $256 \pm 2 \Omega$. Notice that the highest voltage applied in the above measurements is $5 \mathrm{~V}$ dc. Thus, the maximum power consumption for each axis is less than $60 \mathrm{~mW}$ if we take into consideration the temperature coefficient of the platinum resistivity.

\section{Dynamic Measurement Results}

The electromechanical response time of the actuator was measured by monitoring the light spot reflected by the mirror plate with a photosensitive device (PSD). A $2 \mathrm{~Hz}$ square-wave voltage (0-3.5 V) was applied to a single actuator. The voltage output from the PSD shows that the $10 \%$ to $90 \%$ response time is less than $20 \mathrm{~ms}$, as shown in Fig. 7.

The frequency response of the mirror plate was also obtained with the PSD and an SRS 785 signal analyzer. For the piston mode measurement, a same $1 \mathrm{~V}$ dc superimposed by a $100 \mathrm{mV}$ ac signal from the signal analyzer was applied to all four actuators. The light spot reflected by the mirror plate was monitored by the PSD whose output was fed back to the signal analyzer. For the rotation mode measurement, the same setup was used but the ac signal from the signal analyzer was inverted to make a differential pair and then applied to the two opposing actuators. The recorded resonance frequencies for piston and rotation modes are $664 \mathrm{~Hz}$ and $714 \mathrm{~Hz}$, respectively.

One-dimensional (1D) line scans were generated by exciting one pair of the actuators, as shown in Fig. 8(a). Two-dimensional (2D) scans using this device were demonstrated by simultaneously exciting two pairs of the actuators. By varying the frequency and phase of the ac signals, different Lissajous patterns were generated and shown in Fig. 8(b)-(d). It was noticed that when the device was driven at its resonance frequency for the rotation mode, the optical scan angle can go up to $\pm 30^{\circ}$ at $2.25 \mathrm{~V}$ differential voltages.

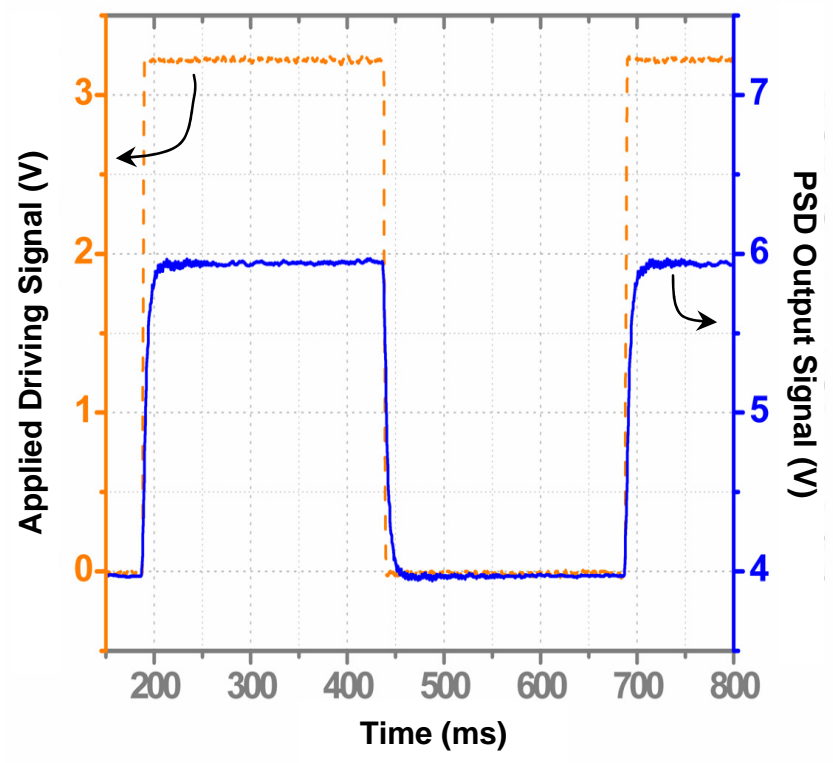

Fig. 7 Electromechanical transient response time measurement. 

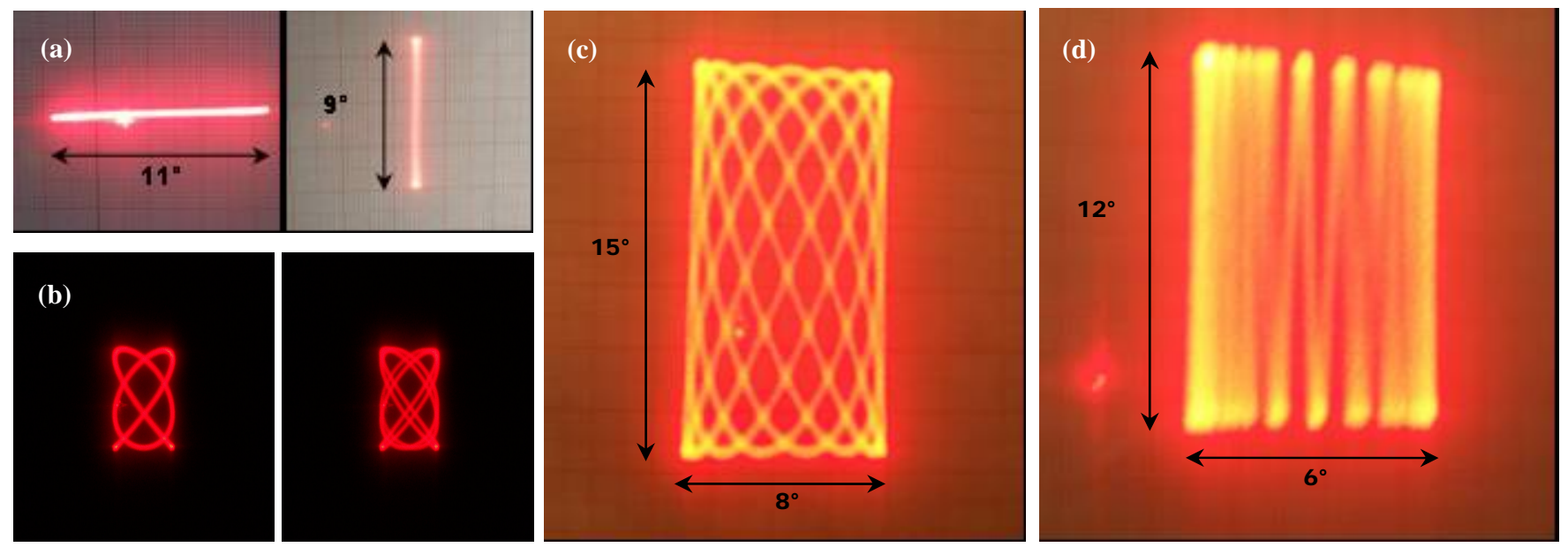

Fig. 8 Various Lissajous patterns generated by varying AC signal frequency ratio and phase difference between the two orthogonal actuator pairs. (a) 1-D scan for $-x$ and $-y$ axis; (b) Lissajous patterns with frequency ratio of 2:3 and a phase difference between two orthogonal actuator pairs; (c) Lissajous patterns with frequency ratio of 5:6; (d) Raster scanning pattern with frequency ratio of 1:9.

\section{Mirror surface quality measurement}

The mirror surface quality was measured using a Wyko-Vision32 optical profilometer. The measured radius of curvature over the $1 \mathrm{~mm}$ by $1 \mathrm{~mm}$ mirror plate surface is $0.32 \mathrm{~m}$ with peak to valley deformation of $0.15 \mu \mathrm{m}$. The surface roughness is about $20 \mathrm{~nm}$.

\section{CONCLUSION}

An agile tip-tilt-piston micromirror with $1 \mathrm{~mm}$ by $1 \mathrm{~mm}$ aperture size has been successfully demonstrated. The mirror can achieve a maximum $141 \mu \mathrm{m}$ piston stroke and $\pm 12^{\circ}$ of $2 \mathrm{D}$ non-resonant optical scan range all at less than $6 \mathrm{~V}$ dc. This design solves the lateral-shift problem for the piston motion. The axis of rotation is also structurally fixed for the 2D scanning mode. The measured electromechanical response time is less than $20 \mathrm{~ms}$. The device has performed various Lissajous patterns successfully. A study on the dynamic deformation of the mirror plate during linear and angular scanning is currently ongoing. Raster scanning for optical displays and optical imaging will be explored in the near future.

\section{ACKNOWLEDGEMENT}

This project is supported by the National Science Foundation under award numbers CBET-0423557 and ECCS-0725598.

\section{REFERENCES:}

[1] K. E. Perterson, "Silicon torsional scanning mirror," IBM Journal of Research and Developmen, 24, pp. 631 (1980).

[2] P. F. V. Kessel et al., “A MEMS-based project display,” Proceedings of IEEE, 86, pp. 1687 (1988).

[3] U. Krishnamoorthy, et al, "Dual-mode micromirrors for optical phase array applications," Sensors and Actuators A, 97-98C, pp. 22-26 (2002).

[4] J. B. Stewart et al., "Open-loop control of a MEMS deformable mirror for large- amplitude wavefront control," J. Opt. Soc. Am. A, 24, pp. 3827-3833 (2007).

[5] J. P. Yang, X. C. Deng, and T. C. Chong, “An electro-thermal bimorph-based micro actuator for precise track-positioning of optical disk drives,” J. Micromech. Microeng., 15, pp. 958965 (2005).

[6] A. D. Yalcinkaya et al., "Two Axis Electromagnetic Microscanner for High Resolution Displays,” JMEMS, 15, 4, pp. 786-794 (2006).

[7] V. Milanovic, K. Castelino, and D. McCormick, "High adaptable MEMS-based display with wide projection angle,” MEMS'07, pp. 143-146 (2007).

[8] J. Tsai, and M. C. Wu, "Design, fabrication, and characterization of a high fill-factor, large scan-angle, two-axis scanner array driven by a leverage mechanism,” JMEMS, 15, 5, pp. 1209- 1213 (2006).

[9] W. Piyawattanametha et al., "Three-dimensional in vivo real time imaging by a miniature dual-axes confocal microscope based on a two-dimensional MEMS scanner," Transducers'07, pp. 439-442 (2007).

[10] V. Milanovic, G. A. Matus, and D. McCormick, “Gimbal-less Monolithic Silicon Actuators for Tip-Tilt-Piston Micromirror Applications," Journal of Selected Topics in Quantum Electronics, 10, 3, pp. 462-471 (2004).

[11] I. W. Jung, U. U. Krishnamoorthy, and O. Solgaard, "High fill-factor two-axis gimbaled tip-tilt-piston micromirror array actuated by self-aligned vertical electrostatic combdrive," JMEMS, 15, 3, pp. 563-571 (2006).

[12] J. B. Stewart et al., "Design and development of a 331segment tip-tilt-piston mirror array for space-based adaptive optics," Sensor and Actuators A, 138, pp. 230-238 (2007).

[13] F. Pardo et al., "Flexible fabrication of large pixel count piston-tip-tilt mirror arrays for fast spatial light modulators," Microelectronic Engineering, 8, pp. 1157-1161 (2007).

[14] J. Singh et al., "A two axes scanning SOI MEMS micromirror for endoscopic bioimaging,” J. Micromech. Microeng., 18, In Press (2008).

[15] A. Jain and H. Xie, “A single-crystal silicon micromirror for large bi-directional 2D scanning applications,” Sensors and Actuators A, 130, pp. 454-460 (2006).

[16] L. Wu and H. Xie, "A large-aperture piston-tip-tilt micromirror for optical phase array applications," MEMS'08, pp. 754-757 (2008).

[17] S. Todd et al., “A multi-degree-of-freedom micromirror utilizing inverted-series-connected bimorph actuators," J. Opt. A: Pure Appl. Opt. 8, pp. 352-359 (2006).

[18] K. Jia and H. Xie, "Multi-axis electrothermal scanning micromirror with low driving voltage," CLEO'07, Nanophotonic Structures and Devices, CThJJ (2007). 